\title{
BMJ Open Study protocol for endoscopic ultrasonography-guided ethanol injection therapy for patients with pancreatic neuroendocrine neoplasm: a multicentre prospective study
}

\author{
Kazuyuki Matsumoto (D) , ${ }^{1}$ Hironari Kato, ${ }^{1}$ Masayuki Kitano, ${ }^{2}$ Kazuo Hara, ${ }^{3}$ \\ Masaki Kuwatani (D) , ${ }^{4}$ Reiko Ashida, ${ }^{2}$ Mamoru Takenaka, ${ }^{5}$ Tatsuhiro Yamazaki, ${ }^{1}$ \\ Jun Sakurai, ${ }^{6}$ Michihiro Yoshida, ${ }^{6}$ Hiroyuki Okada ${ }^{1}$
}

To cite: Matsumoto $\mathrm{K}$, Kato $\mathrm{H}$, Kitano M, et al. Study protocol for endoscopic ultrasonographyguided ethanol injection therapy for patients with pancreatic neuroendocrine neoplasm: a multicentre prospective study. BMJ Open 2021;11:e046505. doi:10.1136/ bmjopen-2020-046505

- Prepublication history for this paper is available online. To view these files, please visit the journal online (http://dx.doi. org/10.1136/bmjopen-2020046505).

Received 01 November 2020 Accepted 08 June 2021

Check for updates

(C) Author(s) (or their employer(s)) 2021. Re-use permitted under CC BY-NC. No commercial re-use. See rights and permissions. Published by BMJ.

For numbered affiliations see end of article.

Correspondence to Dr Kazuyuki Matsumoto; matsumoto.k@okayama-u.ac.jp

\section{ABSTRACT}

Introduction The management of small pancreatic neuroendocrine neoplasms (PNENs) remains controversial. The standard treatment for PNENs is surgical resection; however, invasiveness of surgical procedure remains higher and the incidence of postoperative adverse events is still high. Recently, the efficacy and safety of endoscopic ultrasonography (EUS)-guided ethanol injection for small PNENs has been preliminarily demonstrated. Thus, a multicentre prospective study is being conducted to evaluate the efficacy and safety of EUS-guided ethanol injection therapy for small PNENs.

Methods and analysis The major eligibility criteria are the presence of pathologically diagnosed grade (G) 1 tumour, a tumour size of $\leq 15 \mathrm{~mm}$ and non-functional PNEN or insulinoma. For treatment, we will use a 25 -gauge needle and pure ethanol. Contrast-enhanced CT (CE-CT) will be performed on postoperative day $3-5$, and if enhanced areas of the tumour are still apparent, an additional session is scheduled during the same hospitalisation period. We set the total amount of ethanol per session to $2 \mathrm{~mL}$. To evaluate the efficacy and safety, CE-CT will be performed at 1 and 6 months after treatment. The primary endpoint is the percentage of subjects who achieved all of the following evaluated points. Efficacy will be evaluated based on the achievement of complete ablation (defined as no enhanced area within the tumour on CE-CT) at 1 and 6 months. Safety will be evaluated based on the avoidance of severe adverse events within 1 month after treatment, continuing severe pancreatic fistula at 1 month after treatment and the incidence and/or exacerbation of diabetes mellitus at 6 months after treatment.

Ethics and dissemination This protocol has been approved by Okayama University Certified Review Board (approval number. CRB19-007). The results will be submitted to peer-reviewed journals and will be presented at international conferences.

Trial registration number jRCTs061200016.

\section{INTRODUCTION}

Pancreatic neuroendocrine neoplasms (PNENs) are fairly rare, accounting for

\section{Strengths and limitations of this study}

This is the first prospective multicentre confirmatory clinical study conducted to investigate the efficacy and safety of an endoscopic ultrasonography (EUS)-guided ethanol injection therapy in patients with small grade 1 pancreatic neuroendocrine neoplasms (PNENs).

- This proposed study investigates EUS-guided therapy as a less invasive treatment approach to the treatment of small PNENs in patients without lymph node metastasis in comparison to surgical treatment in terms of both efficacy and safety.

- This study was conducted in a single arm with short-term follow-up period. The follow-up period was not sufficient to assess the efficacy in preventing tumour recurrence after ethanol injection.

$1 \%-2 \%$ of primary pancreatic malignancies. ${ }^{1}$ However, the incidence of PNENs has increased substantially because of the widespread use of advanced endoscopic and radiological imaging techniques. ${ }^{2}$

The treatment methods for PNENs depend on the hormone-related symptoms and tumour size. ${ }^{34}$ For patients with symptomatic disease or tumours of $>2 \mathrm{~cm}$ in diameter, surgical resection is the standard of care. However, the optimal treatment approach for patients with non-functional, small PNENs ( $\leq 2 \mathrm{~cm}$ in diameter) is controversial. Sadot et at reported the outcomes of observation versus resection for small asymptomatic PNENs. In their report, the observation was recommended for 104 patients (observation group), and these patients were matched to 77 patients in a resection group based on tumour size on initial imaging (the median tumour size in the observation and resection 
groups was $12 \mathrm{~mm}$ and $14 \mathrm{~mm}$, respectively). The 5-year overall survival rate of the observation and resection groups were $99 \%$ (95\% CI: $95 \%$ to $100 \%$ ) and $91 \%(95 \%$ CI: $84 \%$ to $97 \%$ ), respectively with a median follow-up period of 44 months and 57 months, respectively; There were no significant differences between the two groups (log-rank, $\mathrm{p}=0.3$ ), and no patients in either group died from the disease. However, within the observation group, 26 of the 104 patients (25\%) underwent subsequent tumour resection after a median observation interval of 30 months (range, 7-135 months) (patient's preference, $\mathrm{n}=10(38 \%)$; increasing tumour size, $\mathrm{n}=8(31 \%)$; physician's preference, $\mathrm{n}=7$ (27\%); and development of pancreatic duct dilatation, $\mathrm{n}=1(4 \%))$. Although observation for stable, small, incidentally discovered PNEN is considered reasonable for selected patients, it is unknown whether a tumour will be aggressive or not when it is small in size. Moreover, patients undergoing observation should be followed up at least once a year using CT or MRI with contrast medium.

The benefit of surgery must be balanced against potential postoperative complications. ${ }^{3-5}$ In a study of surgical resection for benign pancreatic tumours, the morbidity rates for pancreaticoduodenectomy (PD), distal pancreatectomy (DP) and parenchyma-preserving resection were reported to be $52 \%, 47 \%$ and $44 \%$, respectively. ${ }^{5}$ Furthermore, $18 \%$ of patients developed diabetes mellitus (DM) after PD and 14\% developed DM after DP. ${ }^{5}$ These are just some of the problems associated with pancreatic surgery that leads to lower quality-of-life of the patient.

Recently, endoscopic ultrasonography (EUS)-guided ethanol ablation has been proposed for the treatment of patients with small PNENs who refuse surgery or who are poor surgical candidates. ${ }^{6-15}$ Using pure ethanol or ethanol-lipiodol emulsion, ${ }^{7}$ the complete ablation rate has been reported to be approximately $50 \%$ with a single session and up to $60 \%-80 \%$ with an additional session. ${ }^{6-8}$ As for complications, mild pancreatitis occurred in a few patients required a large amount of ethanol $(>2 \mathrm{~mL})$ in one session even if the tumour size was less than $2 \mathrm{~cm} .{ }^{6-15}$ We previously described the efficacy and safety of scheduled early EUS-guided ethanol reinjection therapy for small PNENs. ${ }^{8}$ A total of five patients with pathologicallyproven grade $(\mathrm{G}) 1$ PNENs were treated; the median diameter of target lesion was $10 \mathrm{~mm}$ (range: $7-14 \mathrm{~mm}$ ). Of these five patients, three underwent an additional session at 3 days after the first treatment. The median volume of ethanol injected per session was $0.8 \mathrm{~mL}$ (range: $0.3-1 \mathrm{~mL}$ ), and the mean total volume was $1 \mathrm{~mL}(0.9-1.8 \mathrm{~mL})$. Complete ablation (defined based on contrast-enhanced (CE)-CT at 1 month after treatment) was achieved in four of the five tumours $(80 \%)$. During 1 year of follow-up, none of the patients reported any procedure-related adverse events, including the development of DM, and no patients showed tumour recurrence. The results indicated that the use of a small amount of ethanol reduced the procedure-related adverse events, and that scheduled injection increased the complete ablation rate.
The advantages of EUS-guided therapy are that the reduction of complications can be expected in comparison to surgical resection and that the pancreatic exocrine and endocrine functions can be preserved. On the other hand, EUS-guided therapy is associated with a limitation. Complete tumour ablation is determined based on thin slice CE-CT findings; however, it is sometimes difficult to demonstrate complete tumour elimination. Furthermore, long-term follow-up is required to prove the efficacy of EUS-guided treatment, and lymph node dissection cannot be performed. Thus, EUS-guided treatment should be limited to patients with a low risk of lymph node metastasis and small tumour size.

Hashim et $a l^{16}$ reported on the rate of lymph node metastasis of PNENs according to tumour size. They evaluated 136 patients who underwent surgical resection of PNENs with lymph node resection (surgical methods: DP, $54 \%$; PD, $41 \%$; total pancreatectomy, 2\%; nuclear excision, $2 \%$ ). The lymph node metastasis rates were $12 \%$ $(2 / 17)$ in patients with a tumour diameter of $\leq 10 \mathrm{~mm}$, $14.3 \%(3 / 22)$ in patients with a tumour diameter of $11-15 \mathrm{~mm}$ and $40 \%(8 / 21)$ in patients with a tumour diameter of $16-20 \mathrm{~mm}$. A logistic regression analysis was performed to determine factors associated with the frequency of lymph node metastasis: the frequency was higher in patients with larger tumours $(>1.5 \mathrm{~cm} ; \mathrm{OR}=4.7)$, tumours of the head (in comparison to pancreatic bodytail $(\mathrm{OR}=2.8))$, tumours with $\mathrm{Ki}-67$ of $>20 \% \quad(\mathrm{OR}=6.7)$ and tumours with lymphovascular invasion $(\mathrm{OR}=3.6)$ $(\mathrm{p}<0.05)$. Considering the risk of lymph node metastasis, tumour size $\leq 15 \mathrm{~mm}$ and pathologically G1 are suitable for EUS-guided therapy.

Therefore, we launched the current trial to assess the efficacy and safety of EUS-guided ethanol injection therapy for PNENs of $<15 \mathrm{~mm}$ in size with a pathological diagnosis of G1.

\section{METHODS AND ANALYSIS \\ Study setting}

This study is conducted as a multicentre, single-arm, prospective, phase II study in six high-volume medical centres in Japan. Eligible patients with a PNEN will be treated by EUS-guided ethanol injection and followed up until 6 months after treatment. An overview of the protocol is shown in figure 1 .

\section{Endpoints}

\section{Primary endpoint}

This study establishes a composite primary endpoint. The primary endpoint is the percentages of subjects who achieve all of the following evaluated points.

1. Efficacy:

- Complete ablation on CE-CT at 1 and 6 months after treatment

2. Safety:

- Avoidance of severe adverse events within 1 month after treatment 


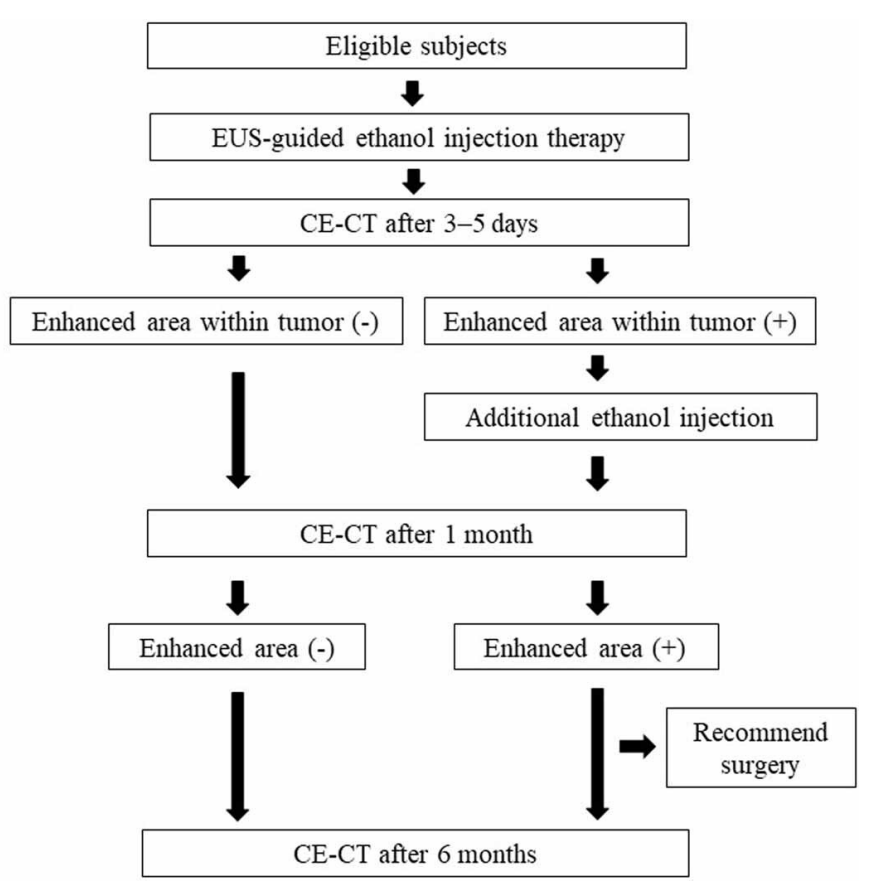

Figure 1 A flow chart of the present study. CE-CT, contrastenhanced CT; EUS, endoscopic ultrasonography.

- Avoidance of severe pancreatic fistula at 1 month after treatment

- Avoidance of the incidence and/or exacerbation of $\mathrm{DM}$ at 6 months after treatment

\section{Secondary endpoints}

The following secondary endpoints will be evaluated in two cohorts: Cohort 1 (tumour size $\leq 10 \mathrm{~mm}$ ) and Cohort 2 (tumour size $10-15 \mathrm{~mm}$ ).

1. Safety:

- Prevalence of total adverse events

- Prevalence of device failures

- Prevalence of conversion to surgery

- Prevalence of severe adverse event within 1 month after procedure

- Prevalence of severe pancreatic fistula at 1 month after procedure

- Prevalence of DM exacerbation at 6 months after treatment.

2. Efficacy:

- Complete ablation on CE-CT at 1 month after treatment

- Complete ablation on CE-CT at 6 months after treatment

- Six-month overall survival.

The following endpoints will only be evaluated in patients with insulinoma.

1. Improvement of hypoglycaemic symptoms

2. Changes in serum levels of fasting blood glucose, insu-

lin and $\mathrm{C}$ peptide from before to after the procedure

\section{Definition}

Complete ablation is defined as the absence of enhanced areas within the tumour on arterial phase CE-CT imaging
Box 1

Inclusion criteria

- Age 20-75 years.

- Patient who has given their fully informed consent.

- A diagnosis of a pancreatic neuroendocrine neoplasm (PNEN) with grade 1 confirmed pathologically by endoscopic ultrasonographyguided fine needle aspiration specimen (WHO 2017 classification).

- Tumour diameter $\leq 15 \mathrm{~mm}$ and well-enhanced tumour in the arterial phase on contrast enhanced-CT (CE-CT).

- A PNEN diagnosed as a non-functional tumour or an insulinoma.

\section{Exclusion criteria}

- Allergy to contrast media or ethanol.

- The distance between tumour and main pancreatic duct is $\leq 2 \mathrm{~mm}$ on radiographic images.

- Suspicion of lymph node metastasis or distant metastasis on CE-CT.

- Prothrombin time $\leq 50 \%$ or international normalised ratio $\geq 1.5$.

- Platelet $\leq 50 \times 10^{9} / \mathrm{L}$.

- Estimated glomerular filtration rate $\leq 30 \mathrm{~mL} / \mathrm{min}$.

- Administered $\geq 2$ antithrombotic agents.

- Performance status $\geq 2$.

- Being pregnant or possibly pregnant.

- Poor prognosis ( $<5$ years) predicted.

- Patients judged as inappropriate candidates by the chief medical examiner.

with a slice thickness of 1-2 $\mathrm{mm}$. The CE-CT images will be reviewed independently by two expert gastroenterologists. If it is difficult to make a judgement by CE-CT, CE-EUS will be performed to assess the existence of enhanced areas within the tumour. Procedure-related adverse events will be evaluated based on American Society for Gastrointestinal Endoscopy (ASGE) guideline 2010, ${ }^{17}$ and other adverse events will be evaluated based on Common Terminology Criteria for Adverse Event (CTCAE) V.5.0. Severe adverse events are defined as moderate or higher in ASGE and grade 3 or higher in CTCAE. Severe pancreatic fistula is defined as continuing any treatment for pancreatic fistula (percutaneous or endoscopic drainage tube and/or medication) at 1 month after the procedure. DM is defined as a fasting blood glucose level of $126 \mathrm{mg}$ / $\mathrm{dL}$ or an occasional blood glucose level of $200 \mathrm{mg} / \mathrm{dL}$ and glycated haemoglobin (HbA1c) $\geq 6.5$ (National Glycohemoglobin Standardisation Programme value). New-onset DM means that a patient without DM at the time of registration newly developed DM, and the exacerbation of DM means that a patient who did not meet the definition for DM at the time of registration starts or adds a medication for DM due to poor glycaemic control, or HbAlc is increased up to $0.2 \%$.

\section{Patient eligibility}

The eligibility and exclusion criteria are shown in box 1. EUS-guided fine needle aspiration (FNA) will be performed to exclude the possibility of lymph node metastasis. A flow chart of suspicion of lymph node metastasis on CE-CT is shown in figure 2. 


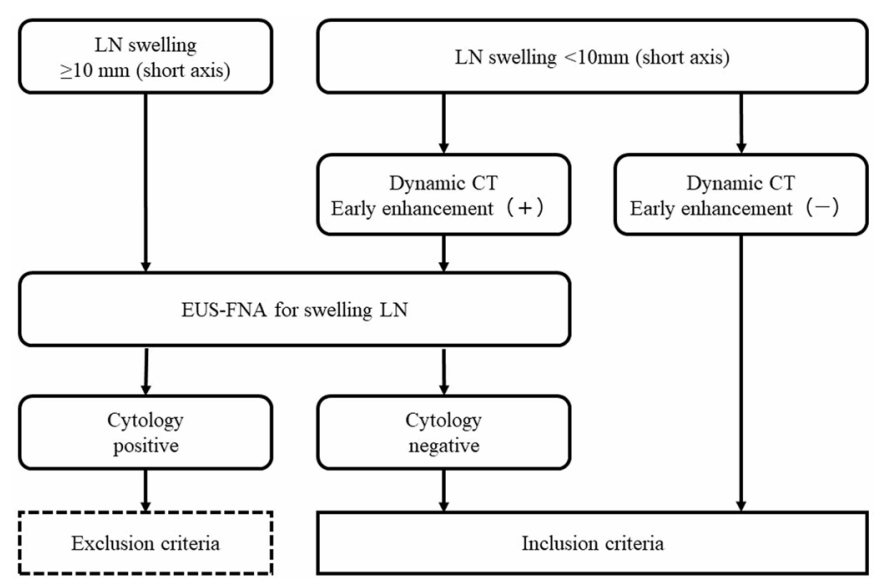

Figure 2 A flow chart of the suspicion of lymph node metastasis on CE-CT. CE-CT, contrast-enhanced CT; EUSFNA, endoscopic ultrasonography-guided fine needle aspiration; LN, lymph node.

\section{Study procedure}

The procedure is performed with the patient in a prone or semi-prone position under conscious sedation using intravenous anaesthetic in the endoscopy room. For treatment, a 25-G fine-needle aspiration needle (EZ-shot 3; Olympus Medical Systems, Tokyo, Japan) filled with ethanol is advanced into the tumour under EUS. Then, pure ethanol (Mylan Seiyaku, Tokyo, Japan) is injected until a hyperechoic blush extends to the edge of the tumour margin and the needle is kept inside the tumour for at least $1 \mathrm{~min}$ to avoid the backflow of ethanol. Once the needle is removed, we check for low-echoic areas of the tumour. If such areas are detected, ethanol is added to the site. For the sake of safety, the amount of ethanol per puncture is set to $1 \mathrm{~mL}$, the total number of punctures per session is set to 3 , and the maximum volume of ethanol per session is set to $2 \mathrm{~mL}$.

At 3-5 days after the treatment, CE-CT is performed to evaluate the tumour viability and procedure-related adverse events. If enhanced areas of the tumour are noted on postprocedural CE-CT, we will perform an additional ablation session during the same hospitalisation period. The patient is discharged 1 day after this additional session. If there are no enhanced areas of the tumour on postprocedural CE-CT, then the patient is discharged. Blood testing is performed at 2 hours postoperatively and on postoperative day 1 .

\section{Follow-up}

Follow-up examinations are scheduled for 1, 3 and 6 months to evaluate the general condition of the patient, and blood testing is performed. The patients are also scheduled to undergo follow-up CE-CT imaging at 1 and 6 months after discharge. When incomplete ablation of the treated lesion is judged to have occurred based on follow-up CE-CT, salvage surgical resection will be considered to the patient.
Patient and public involvement

No patient involved.

\section{Sample size calculation}

A historical data set was analysed to evaluate surgical treatment in 23 patients with PNEN ( $\leq 15 \mathrm{~mm}$ in diameter) who underwent treatment at Okayama University Hospital from November 2007 to January 2018. This data showed that $48 \%(11 / 23)$ of the patients met the composite primary endpoints; the local control rate for target lesion was $100 \%(23 / 23)$, the rate of severe adverse events (Clavien-Dindo grade III-IV) within 1 month after treatment was $35 \%(8 / 23)$, the rate of severe pancreatic fistula at 1 month after treatment was $35 \%(8 / 23)$ and the incidence of DM or the exacerbation of DM at 6 months after treatment was $17 \%$ (4/23), respectively. In our previous pilot study of EUS-guided ethanol injection therapy, $75 \%(6 / 8)$ of the patients achieved the composite primary endpoints; the complete ablation rate at 1 and 6 months was $75 \%(6 / 8)$, no patients experienced severe adverse events, no patients developed severe pancreatic fistula and no patients developed DM or experienced an exacerbation of DM. Thus, the null and alternative hypotheses are given below:

$$
\begin{aligned}
& \mathrm{H}_{0}: \mathrm{P}_{\mathrm{T}}=0.48 \\
& \mathrm{H}_{1}: \mathrm{P}_{\mathrm{T}}=0.75
\end{aligned}
$$

where $\mathrm{P}_{\mathrm{T}}$ is the anticipated composite primary endpoint after the study procedure. Against abovementioned background, 22 patients would provide a statistical power of $80 \%$ to test the primary hypothesis at the two-sided significance level of 0.1. Considering non-compliance and/or dropout patients, 25 patients are recruited in total.

\section{Statistical analysis}

The data analysis is performed according to an intentionto-treat principle. Clopper-Pearson's exact binomial test and CI estimation are applied for the primary endpoint and the secondary endpoints. The statistical significance level is set at $10 \%$ for the purpose of orphan disease regulatory filing, otherwise $5 \%$ (two-sided). All analyses are conducted with SAS software (SAS Institute).

\section{ETHICS AND DISSEMINATION}

Written informed consent from all the patients screened will be obtained before the procedures start. The study protocol has been approved by Okayama University Certified Review Board (approval number. CRB19-007) and registered in Japan Registry of Clinical Trial. Monitoring and auditing will be carried out throughout the trial. We will also establish an independent data monitoring committee consisting of three additional doctors who were not associated with the study to determine whether or not the study should continue if severe adverse events occur.

\section{Author affiliations}

${ }^{1}$ Department of Gastroenterology and Hepatology, Okayama University Hospital, Okayama, Japan 
${ }^{2}$ Second Department of Internal Medicine, Wakayama Medical University,

Wakayama, Japan

${ }^{3}$ Gastroenterology, Aichi Cancer Center, Nagoya, Japan

${ }^{4}$ Gastroenterology and Hepatology, Hokkaido University Hospital, Sapporo, Japan

${ }^{5}$ Department of Gastroenterology and Hepatology, Kindai University, Osaka-Sayama, Japan

${ }^{6}$ Center for Innovative Clinical Medicine, Okayama University Hospital, Okayama, Japan

Contributors $\mathrm{KM}, \mathrm{HK}$ and JS: the conception and design of the research and writing the paper. MKi, KH, MKu, RA and MT: contributed to the background and discussion check. TY, JS and MY: contributed to the statistical analysis. HO: final approval of the article. All authors read and approved the final manuscript.

Funding The authors have not declared a specific grant for this research from any funding agency in the public, commercial or not-for-profit sectors.

Competing interests None declared.

Patient and public involvement Patients and/or the public were not involved in the design, or conduct, or reporting, or dissemination plans of this research.

Patient consent for publication Not required.

Provenance and peer review Not commissioned; externally peer reviewed.

Open access This is an open access article distributed in accordance with the Creative Commons Attribution Non Commercial (CC BY-NC 4.0) license, which permits others to distribute, remix, adapt, build upon this work non-commercially, and license their derivative works on different terms, provided the original work is properly cited, appropriate credit is given, any changes made indicated, and the use is non-commercial. See: http://creativecommons.org/licenses/by-nc/4.0/.

ORCID iDs

Kazuyuki Matsumoto http://orcid.org/0000-0002-5102-7452

Masaki Kuwatani http://orcid.org/0000-0002-0265-6068

\section{REFERENCES}

1 Yao JC, Eisner MP, Leary C, et al. Population-based study of islet cell carcinoma. Ann Surg Oncol 2007:14:3492-500.

2 Ito T, Sasano H, Tanaka M, et al. Epidemiological study of gastroenteropancreatic neuroendocrine tumors in Japan. $J$ Gastroenterol 2010;45:234-43.
3 Falconi M, Eriksson B, Kaltsas G, et al. ENETS consensus guidelines update for the management of patients with functional pancreatic neuroendocrine tumors and non-functional pancreatic neuroendocrine tumors. Neuroendocrinology 2016;103:196-211.

4 Sadot E, Reidy-Lagunes DL, Tang LH, et al. Observation versus resection for small asymptomatic pancreatic neuroendocrine tumors: a matched case-control study. Ann Surg Oncol 2016;23:1361-70.

5 Falconi M, Mantovani W, Crippa S, et al. Pancreatic insufficiency after different resections for benign tumours. Br J Surg 2008;95:85-91.

6 Park DH, Choi J-H, Oh D, et al. Endoscopic ultrasonography-guided ethanol ablation for small pancreatic neuroendocrine tumors: results of a pilot study. Clin Endosc 2015;48:158-64

7 Choi J-H, Park DH, Kim M-H, et al. Outcomes after endoscopic ultrasound-guided ethanol-lipiodol ablation of small pancreatic neuroendocrine tumors. Dig Endosc 2018;30:652-8.

8 Matsumoto K, Kato H, Kawano S, et al. Efficacy and safety of scheduled early endoscopic ultrasonography-guided ethanol reinjection for patients with pancreatic neuroendocrine tumors: prospective pilot study. Dig Endosc 2020;32:425-30.

9 Jürgensen C, Schuppan D, Neser F, et al. EUS-guided alcohol ablation of an insulinoma. Gastrointest Endosc 2006;63:1059-62.

10 Muscatiello N, Salcuni A, Macarini L, et al. Treatment of a pancreatic endocrine tumor by ethanol injection guided by endoscopic ultrasound. Endoscopy 2008;40(Suppl 2):E258-9.

11 Deprez PH, Claessens A, Borbath I, et al. Successful endoscopic ultrasound-guided ethanol ablation of a sporadic insulinoma. Acta Gastroenterol Belg 2008;71:333-7.

12 Vleggaar FP, Bij de Vaate EA, Valk GD, et al. Endoscopic ultrasoundguided ethanol ablation of a symptomatic sporadic insulinoma. Endoscopy 2011;43(Suppl 2 UCTN):E328-9.

13 Levy MJ, Thompson GB, Topazian MD, et al. US-guided ethanol ablation of insulinomas: a new treatment option. Gastrointest Endosc 2012;75:200-6.

14 Qin S-Y, Lu X-P, Jiang H-X. EUS-guided ethanol ablation of insulinomas: case series and literature review. Medicine 2014;93:e85

15 Lakhtakia S. Therapy of pancreatic neuroendocrine tumors: fine needle intervention including ethanol and radiofrequency ablation. Clin Endosc 2017;50:546-51.

16 Hashim YM, Trinkaus KM, Linehan DC, et al. Regional lymphadenectomy is indicated in the surgical treatment of pancreatic neuroendocrine tumors (PNETs). Ann Surg 2014;259:197-203.

17 Cotton PB, Eisen GM, Aabakken L, et al. A lexicon for endoscopic adverse events: report of an ASGE workshop. Gastrointest Endosc 2010;71:446-54. 